\title{
Transparencia, paz y sostenibilidad. Una aproximación latinoamericana
}

\author{
ENOC ELISEO MOJICA-SAUZA* \\ FLORINA GUADALUPE ARREDONDO-TRAPERO** \\ JOSÉ CARLOS VÁZQUEZ-PARRA***
}

\begin{abstract}
RESUMEN El presente artículo se enfoca en la relevancia que tienen las empresas para el desarrollo de entornos que procuren la transparencia, la sostenibilidad y la paz, considerando todos sus grupos de interés tanto por medio de acciones internas como a partir de la generación de redes de colaboración intersectorial. Por medio de una reflexión de carácter teórico se pretende plantear a las organizaciones como actores sociales estratégicos en la construcción de la paz y la sostenibilidad de la región. En un inicio se aborda el rol que desempeñan las organizaciones como entes sociales transparentes, al igual que agentes promotores de sostenibilidad y paz. Posteriormente se ve la necesidad de participación de organismos intersectoriales, así como se señalan ejemplos de programas organizacionales que tienen por objetivo la transparencia empresarial y la participación en situaciones de posconflicto. El mundo, por la globalización y la interconectividad, requiere de la participación de todos los actores sociales para la construcción de un futuro sostenible. Para ello se necesitan nuevas formas de pensar y actuar para enfrentar los retos de paz y sostenibilidad. Más allá, se señala la necesidad de un cambio de carácter cultural que facilite una visión amplia y transparente para construir nuevos escenarios de colaboración.
\end{abstract}

COMO CITAR ESTE ARTÍCULO: How to cite this article:

Mojica-Sauza, E.E., ArredondoTrapero, F.G. y Vázquez-Parra, J.C. (2019). Transparencia paz y sostenibilidad. Una aproximación latinoamericana. Revista Perspectiva Empresarial, 6(1), 111-127

Recibido: 16 de mayo de 2018 Aprobado: 22 de octubre de 2018

PALABRAS CLAVE redes intersectoriales, paz, sostenibilidad, transparencia, corrupción.

\footnotetext{
*Maestroen ProspectivaEstratégica.TecnológicodeMonterrey,NuevoLeón,México.E-mail:ems1985@gmail.com.ORCID:0ooo0002-0344-6468. Google Scholar: https://scholar.google.com/citations?view_op=list_works\&hl=en\&user=nXFTyTAAAAAJ.

** Doctora en Economía y Dirección de Empresas. Profesora Titular. Tecnológico de Monterrey, Nuevo León, México. E-mail: farredon@tec.mx. ORCID: 0000-0003-4909-0329. Google Scholar: https://scholar.google.com/ citations?hl=es\&user=xvopaCgAAAAJ.

*** Doctor en Estudios Humanísticos. Director Departamento de Estudios Humanísticos. Tecnológico de Monterrey, Nuevo León, México. E-mail: jcvazquezp@tec.mx. ORCID: 00oo-0001-9197-7826. Google Scholar: https://scholar.google.com/ citations?hl=es\&user=avmfwbQAAAAJ.
} 


\section{Transparency, peace and sustainability. A Latin American approximation}

ABSTRACT The present paper focuses on the relevance companies have for the development of environments striving for transparency, sustainability and peace, considering all their stakeholders, booth through internal actions as from the generation of intersectoral collaboration networks. By means of a theoretical reflection, the aim of this work is to propose organizations as strategic social actors in the construction of peace and sustainability for the region. Firstly, we deal with the role of organizations as transparent social entities, as well as sustainability and peace promoting agents. Then, a need for the participation of intersectoral bodies is identified, and examples of organizational programs are highlighted which aim at businesses' transparency and participation in post-conflict situations. Due to globalization and interconnectivity, the world requires the participation of all social actors for the construction of a sustainable future. For this, new ways of thinking and acting to face peace and sustainability challenges are needed. Beyond that, the need for a change of a cultural nature is pointed out, one that facilitates a broad and transparent vision to build new collaboration scenarios

KEY WORDS Intersectoral networks, peace, sustainability, transparency, corruption.

Transparência, paz e sustentabilidade. Uma aproximação latino-americana

RESUMO 0 presente artigo se enfoca na relevância que tem as empresas para o desenvolvimento de entornos que procurem a transparência, a sustentabilidade e a paz, considerando todos seus grupos de interesse tanto por meio de ações internas como a partir da geração de redes de colaboração intersectorial. Por meio de uma reflexão de carácter teórico se pretende expor às organizações como atores sociais estratégicos na construção da paz e a sustentabilidade da região. Num início se aborda o papel que desempenham as organizações como entidades sociais transparentes, da mesma forma que agentes promotores de sustentabilidade e paz. Posteriormente se vê a necessidade de participação de organismos intersectoriais, assim como se mostram exemplos de programas organizacionais que tem por objetivo a transparência empresarial e a participação em situações de pós-conflito. 0 mundo, pela globalização e a interconectividade, requere da participação de todos os atores sociais para a construção de um futuro sustentável. Para isso se necessitam novas formas de pensar e atuar para enfrentar os desafios de paz e sustentabilidade. Indo além, se mostra a necessidade de uma mudança de carácter cultural que facilite uma visão ampla e transparente para construir novos cenários de colaboração.

PALAVRAS-CHAVE redes intersectoriais, paz, sustentabilidade, transparência, corrupção. 


\section{Introducción}

Pensar en la paz, y tener la esperanza de un futuro sostenible, es un reto para las democracias del siglo XXI. La idea de paz y sostenibilidad requiere nuevas formas de organización social; requiere de un contexto multilateral que integre el conjunto del gobierno, la empresa y la sociedad como coparticipes del interés por asegurar el bien común.

Esta integración de los distintos actores sociales en la generación de una sociedad de paz y sostenibilidad enfrenta un obstáculo ineludible: la corrupción. La corrupción es una forma de violencia en las relaciones entre los individuos, entre estos y las instituciones. Con los efectos perniciosos que ella genera, la corrupción se ha naturalizado y legitimado de tal forma que pareciera una situación inapelable e imposible de cambiar en los entornos latinoamericanos (Castañeda, 2016). La corrupción y falta de transparencia se ha justificado como un mal necesario que permea en los diversos sectores de la sociedad (Pérez, 2016). La posibilidad de entrever la realidad con mayor transparencia pareciera utópica.

No obstante, a la par de esta forma de pensar, también está la posibilidad de trastocar estos ciclos de opacidad en diversas instancias tal como lo es la vida de las organizaciones y su entorno. Afrontar la corrupción y la violencia que genera a través de la transparencia, abre también la posibilidad de construir la paz desde las organizaciones. Para ello será necesario imaginar la posibilidad de una realidad organizacional diferente.

Este artículo tiene como objetivo analizar la relevancia de la empresa como actor social en la promoción de la transparencia no solo dentro de la empresa como organización, sino también hacia fuera a través de redes de colaboración intersectorial. El texto atiende en primera instancia el planteamiento de la transparencia desde las empresas, presentando el problema de la corrupción, y cómo las organizaciones desde sus prácticas y su cultura pueden ser promotoras de la transparencia en este cambio de paradigma. En una segunda sección se vincula la transparencia empresarial con los conceptos públicos de paz y sostenibilidad. A continuación, se presenta a la empresa como entidad integrada a la res publica a través de redes intersectoriales de colaboración. Finalmente se propone al sector empresarial como un actor estratégico en la construcción de paz y sostenibilidad social.

\section{Empresa como organización social transparente}

\section{La empresa como organización social en un mundo complejo}

Evolutivamente fue la capacidad de organizarse y trabajar colaborativamente lo que permitió a los primeros seres humanos conquistar los espacios geográficos y lograr una adaptación a entornos desconocidos. La relación social favoreció la capacidad de emprender proyectos que les permitieron al hombre y a la mujer satisfacer sus necesidades básicas para la subsistencia.

Hoy en día, la empresa que pretenda asegurar su sostenibilidad debe encontrar la forma de adaptarse a un ambiente cada vez más inestable. Para Arredondo Trapero, De la Garza García y Villa Castaño (2015) la complejidad de las corporaciones se ha ido incrementando, por lo que la necesidad de gestionar la colaboración entre quienes laboran en una organización y entre las propias organizaciones se ha vuelto cada vez más prioritario. Adicionalmente el desarrollo tecnológico ha abierto nuevas posibilidades, pero su dinamismo ha suplido a la estabilidad y ha puesto en riesgo al entorno. En una sociedad del riesgo, las amenazas y las oportunidades se potencializan. Así, es posible afirmar que la empresa experimenta la complejidad y la incertidumbre en la que el viejo paradigma ceteris paribus (todo lo demás constante) ha pasado al mutatis mutandis (todo cambia al mismo tiempo) (Villarreal y Villarreal, 2003).

Por una parte $-\mathrm{y}$ con base en Arredondo Trapero, De la Garza-García y Villa Castaño (2015) - la colaboración fomenta la confianza, ayuda a que la organización innove y logre generar ventajas competitivas. Y por otra, según Etkin (2007), el modelo racional y utilitario se ha vuelto insuficiente para dar respuesta a un entorno 
generalizado de desconfianza y falta de colaboración; la empresa, como organización social, no es ajena a ello.

Bajo este escenario se requiere que las empresas se sustenten en principios que favorezcan la construcción de proyectos compartidos, ya que solo gracias a ello se podrá pensar en satisfacer las demandas de una sociedad que impone desafíos en los que la transparencia se demanda como principio básico para lograr un entorno en paz y sostenible.

\section{La transparencia, la rendición de cuentas y la colaboración en las organizaciones}

Uno de los principios fundamentales para la construcción de proyectos compartidos y de colaboración entre las partes es el de la transparencia. La transparencia puede verse como un atributo que permite una información clara y precisa sobre algo o alguien y que aumenta nuestra capacidad de comprensión, vigilancia y comunicación (Guerrero, 2008). También puede verse como una práctica o instrumento que permite hacer pública la información que facilita la toma de decisiones (Aguilera, 2010). De igual manera la transparencia es una actitud (Marcuello et al., 2007), una disposición de informar (Alonso, 2009), un valor fundamental (Cuevas, 2005) que da apertura y visibilidad de la información y que permite prácticas de certidumbre entre las organizaciones y los entes internos y externos que se relacionan con esta (Uvalle, 2007), al igual que genera credibilidad a quien hace uso de ella (Alejos, 2015).

Aunque muy relacionados es importante distinguir entre el término transparencia, rendición de cuentas y acceso a la información. Por un lado, la rendición de cuentas es una obligación de la empresa de exponer la información que es demandada por un agente específico externo; mientras que la transparencia no responde a una obligación para con un demandante específico sino que es una disposición de poner información en la vitrina pública (Arredondo, De la Garza y Vázquez, 2015). Para distinguir entre los conceptos de transparencia y acceso a la información, Guerrero
(2008) utiliza dos tipos de significado de la transparencia: uno restringido y uno amplio. En el sentido restringido, transparencia refiere a aquella información que por ley las diversas instancias deben exponer de manera pública. En contraste con este sentido restringido de la transparencia, el acceso a la información refiere a la información que debe solicitarse para obtenerla. En el sentido amplio, la transparencia vendría a significar una "política de transparencia"; mientras que el acceso a la información refiere al derecho de acceso a la información (Guerrero, 2008).

Uno de los principales factores en el desempeño de las organizaciones reside en el flujo de la información entre sus miembros. Este flujo puede estar afectado por un mal diseño que impide que la información adecuada esté presente en la toma de decisiones (Vergara, 2005). Siguiendo este razonamiento,

\footnotetext{
la transparencia, por lo tanto, es un facilitador del funcionamiento organizacional porque reduce las probabilidades de que algunos actores organizacionales desvíen su comportamiento de los intereses y objetivos de la organización [...] es un mecanismo que ayuda a que los arreglos colectivos se respeten y a que todos trabajen por un bien común. (Vergara, 2005, p. 27)
}

En la sociedad del conocimiento, los modelos de colaboración interna y externa se han ido especializando; de ahí que la información y su nivel de transparencia sean elementos clave en tal dinámica (Rawlins, 2008). Por ende, el concepto de transparencia es fundamental para la colaboración en y entre las organizaciones.

\section{Implicaciones de la transparencia en el funcionamiento de la empresa}

Las capacidades de organización en toda empresa han permitido la evolución de los sistemas de producción y organización social. La eficiencia dentro de la empresa se ve beneficiada de la organización, la división del trabajo y la coordinación que permite que los diversos agentes contribuyan en favor de mayores beneficios comunes (Schnackenberg and Tomlinson, 2016). 
Esta noción de división de trabajo y especialización, tan importante en la idea de competitividad empresarial moderna, vincula directamente al concepto de transparencia como un diferenciador empresarial intangible que fortalece el capital social e intelectual de una organización (Alejos, 2015). Lo anterior, debido a que la transparencia fomenta la colaboración creativa entre los integrantes de la empresa (Uvalle, 2016).

En la medida en que la demanda por una mejora en la calidad de bienes y servicios se ve satisfecha en contextos colaborativos los niveles jerárquicos dentro de la empresa deberán estar diseñados para la generación de puentes que permitan un flujo de información no solo de arriba abajo, sino también de abajo hacia arriba y en ambos sentidos laterales (Testera y Cabeza, 2013). En ocasiones son los elementos operativos quienes tienen la información clave para mejorar procesos. Estas mejoras apoyan en la reducción de costos, optimizan productos o la experiencia de los empleados o clientes (Bandsuch, Pate and Thies, 2008). Existe una noción de complementariedad que se basa en el reconocimiento y la confianza que existe entre los miembros que integran las relaciones laborares (Giménez, 2012).

La transparencia no solo fundamenta bienes intangibles que fortalecen las relaciones internas a favor de la eficacia y la competitividad sino que también es un elemento clave para la inversión externa. La participación de las empresas en los mercados de valores requiere de un proceso de transparencia que asegure la protección de intereses de accionistas, administradores e inversionistas (Giménez, 2012). Y no es solo la protección sino que la misma intención de inversión externa requiere de un contexto de transparencia que facilite el análisis financiero en beneficio de una mejor toma de decisiones (Guerrero, 2008).

La transparencia ha sido entonces parte del funcionamiento y de la evolución de las empresas competitivas de mercado (del Campo, Herrador y Segovia, 2016). Surge entonces la pregunta: ¿cómo es que los factores de la vida organizacional intervienen en la implementación de una transparencia que ofrece confianza y colaboración en la búsqueda de objetivos compartidos?

Arredondo, De la Garza y Vázquez (2015) realizaron un estudio empírico que evalúa los factores de la vida organizacional que se relacionan con la transparencia y la rendición de cuentas. Los factores evaluados fueron el diálogo, la normatividad, la equidad, la no corrupción interna, la confidencialidad, la integridad y la lealtad.

El estudio demuestra que el diálogo, la lealtad, la equidad y la no corrupción interna son vías directas que favorecen la transparencia, considerando que sin transparencia no hay credibilidad; en este sentido la transparencia dota de legitimidad al valor de la marca y al quehacer de la empresa en la sociedad (Socias y Horrach, 2013). La sostenibilidad de la empresa y de su entorno, fundada en las relaciones con actores internos y externos a la organización, se verá beneficiada por este sentido de legitimidad que otorga una cultura corporativa de transparencia.

\section{La transparencia y su integración a la cultura corporativa}

Una vez que ha sido justificado el valor de la transparencia en las empresas es necesario dejar en claro que esta debe arraigarse no solo a un nivel operativo sino como parte de la cultura organizacional. De acuerdo con Kotter (1996) referir a la cultura en un sentido amplio se entiende como las normas de comportamiento y los valores compartidos dentro de un grupo de personas. Las normas de comportamiento del grupo son las prácticas de acción que se vuelven comunes entre sus integrantes. Estas suelen ser enseñadas como parte del proceso de integración de nuevos miembros, premiando a quienes las adoptan y castigando a quienes no logran adaptarse a ellas (Kotter, 1996).

La cultura organizacional determina la manera en que sus integrantes interpretan la realidad que les rodea. Ante esto se establecen prioridades que definen la toma de decisiones cotidiana. Así, el comportamiento de los individuos termina por tener una relación simbiótica con las formas culturales de las organizaciones a las que pertenecen.

Pensar en el valor de la transparencia como un elemento integrador de una cultura corporativa es pensar en una organización que entiende los objetivos como compartidos, y ese entendimiento moldea de fondo los comportamientos (Manfredi, 
Herranz y Calvo, 2017). Cabe señalar que lograr instaurar valores no es una tarea sencilla. Obtener el apoyo de los líderes organizacionales involucrados es solo el inicio de un proceso de implementación y gestión cotidiana que no se da de manera automática. No es suficiente la buena voluntad, se requieren prácticas que se integren en las rutinas y en los procedimientos cotidianos. Se requiere, además, de una intencionalidad y un verdadero compromiso para apuntalar el empuje de la transparencia a nivel cultural. Especialmente cuando la cultura que se quiere cambiar está cargada de rutinas, procedimientos y estilos de liderazgo que no promueven la transparencia (Briano y Saavedra, 2015). El proceso es complejo e implica asumir el no poder controlarlo del todo.

De acuerdo con Vergara (2005) la implementación del cambio organizacional para mejorar la transparencia se da en dos niveles. En primer lugar está la dimensión técnica, que cambia las normas y procedimientos a través de reglamentos y manuales de operación. En esta dimensión el costo está relacionado con la calidad técnica de la intervención. En un segundo nivel está el proceso de adaptación humana. Por inercia, existirá resistencia por una forma de decidir y actuar más transparente y se buscará hacer las cosas como se tiene por costumbre (Vergara, 2005).

Instaurar el valor de la transparencia implica modificar valores que orientan los comportamientos de los individuos. Se requiere un proceso de incorporación en el que los individuos se comporten con transparencia, no solo por una exigencia de reglamento sino por convencimiento. Si bien en la dimensión racional los cambios deben buscarse como inmediatos, el cambio cultural requiere paciencia y perseverancia. Por esta razón, el cambio por una cultura de transparencia requiere de tiempo (Christensen and Cornelissen, 2015).

Según Kotter (1996) para lograr el cambio organizacional no es suficiente el liderazgo de una sola persona, se requiere un grupo estratégico sólido para desarrollar y comunicar una visión; y posteriormente impulsar y sostener los procesos relacionados a los cambios que de esta derivan. El grupo debe estar consolidado puesto que si no existe compromiso, confianza y objetivos compartidos, este puede incluso ser nocivo a la visión institucional. El grupo debe contar con experiencia, conocimiento, influencia y credibilidad, habilidades de liderazgo y capacidad administrativa para el diseño y la transmisión de objetivos y decisiones racionales y éticas.

La atención a la dimensión humana y cultural como factor reflexivo es lo que permite gestionar las tensiones internas y la incertidumbre del entorno. Una organización sostenible requiere de construir un capital social como filosofía empresarial (Albu and Flycerbom, 2016). Esto demanda que se inserte en la cultura una inteligencia social que integre -basados en la transparencia- relaciones colaborativas, de confianza y compromiso que están en la base de su capital social. Una vez instauradas estas relaciones es necesario cuidar, no degradarlas, y promover círculos virtuosos que favorezcan la sostenibilidad de la empresa y de su entorno (Etkin, 2007).

La gestión transparente se desarrolla en una organización con canales de comunicación interna y externa. Esto es necesario, pues los cambios culturales requieren de individuos integrados a la organización y empoderados para resolver problemas a partir de la información a la que tienen acceso (Ciamberlani y Steinberg, 1999).

Anclar los cambios a nivel cultural, en este caso, a favor de la transparencia, tiene las siguientes cinco premisas: (i) forma parte del proceso de transformación; (ii) convencer de manera clara que la nueva forma de hacer las cosas es mejor que la anterior; (iii) requiere instrucciones verbales y apoyo constante del liderazgo de la empresa; (iv) puede implicar cambiar a personas estratégicas y (v) requiere de incentivos que promuevan las nuevas prácticas (Kotter, 1996).

En resumen, la cultura organizacional favorece la capacidad de difundir y sostener las prácticas y beneficios institucionales de la transparencia. Aunque no es tarea fácil, sí es posible realizar una transformación organizacional que permita una cultura de mejora significativa hacia un entorno más transparente. Los beneficios de ello superan por mucho el esfuerzo invertido en esta empresa. 


\section{La integración del sector empresarial en la construcción de paz y sostenibilidad}

\author{
El problema de la corrupción y sus \\ implicaciones en la vida organizacional
}

La sostenibilidad de la sociedad moderna depende de la integración de actores estratégicos en la búsqueda e implementación de soluciones, y esa integración demanda formas cada vez más transparentes de hacer las cosas (Gilli, 2017).

La paz es un prerrequisito esencial a la hora de enfrentar los retos compartidos en la construcción de sociedades sostenibles. Sin paz es imposible tener la confianza, la cooperación y la inclusión que entre organizaciones se requiere para orquestar soluciones consensuadas. No solo esto, la noción de paz positiva está asociada con características sociales deseables tales como el bienestar, el rendimiento económico, la equidad y el desempeño medioambiental (Institute for Economics and Peace, 2016).

El Instituto para la Economía y la Paz (2015) encontró a través de un estudio que dentro de múltiples modelos, la corrupción es la única variable exploratoria que muestra correlación significativa y consistente con una variedad de indicadores clave de paz y violencia.

Según la información que presenta el índice de paz global (Institute for Economics and Peace, 2016), el costo económico de la violencia en la economía global para el año 2015 fue de US $\$ 13,6$ trillones en términos de paridad del poder adquisitivo -PPA-. Esto representa el 13,3 \% de la actividad económica mundial, es 11 veces el tamaño de la inversión extranjera directa global y es comparable con un costo de US\$ 1876 por cada habitante del mundo (Institute for Economics and Peace, 2016). De esta manera la inversión para atender el tema de la violencia reduce la capacidad de un Estado para orientar sus recursos hacia el bienestar social, lo que genera mayores niveles de violencia.
Es evidente que la corrupción va más allá de la moral individual o empresarial. La corrupción tiene consecuencias sociales que nos afectan a todos (Reyes Heroles, 2007). La corrupción da lugar a sistemas económicos injustos, altera la distribución justa de recursos, desvía fondos, genera beneficios indebidos y afecta las reglas de una competencia justa. Adicionalmente trastoca el Estado de derecho y la cultura de la legalidad, pues se ignoran los derechos legítimos de quienes sufren y reclaman la injusticia de una situación. De este modo la falta de transparencia en las organizaciones oculta mediante dádivas, favores, privilegios o beneficios indebidos, la demanda legítima de quienes se sienten violentados en sus derechos (Spreitzer, 2007).

De acuerdo con Galtung (1996) existen intereses de carácter económico que no solo toleran sino que son generadores de violencia. Esos intereses no son explícitos, pero sutilmente permean en las estructuras de los sistemas. Retomando e interpretando a Galtung (1996) -en el tema de la transparencia en las organizaciones- la corrupción no solo se trata de una forma de violencia cultural, sino también de una forma de violencia estructural.

Por otra parte, desde este tenor, la corrupción (entendida como falta de transparencia u opacidad en la vida de las organizaciones) puede resultar para algunos intereses particulares como un problema conveniente y rentable; aunque sin duda alguna insostenible. Sin embargo ante estas posturas que suelen permear dentro y entre las organizaciones se propone buscar nuevas formas de quehacer organizacional para gestionar relaciones más justas, pacíficas y sostenibles. Repensar la forma de ser de las organizaciones - no como lo que son, sino como debieran ser- requiere de la imaginación ética, el pensar que otra forma de hacer empresa es posible (Lindley, 2007).

Un entorno organizacional más transparente reduce la posibilidad del conflicto. Un conflicto que se intenta evadir mediante la corrupción, se convierte en una situación de violencia que detona de alguna u otra manera en un momento u otro. En el entorno organizacional la falta de transparencia se puede reflejar en: un clima deteriorado; renuncia de personal; negociaciones obstruidas o suspendidas con clientes o proveedores e incluso se puede llegar a los tribunales (Heimstädt, 2017). 
Además de las implicaciones humanas, todas estas consecuencias de la falta de transparencia comprometen la viabilidad de las organizaciones en el largo plazo y alteran la dinámica del sistema económico en su conjunto (Gilli, 2017). En otras palabras, la transparencia se legítima desde las relaciones pacíficas entre el individuo y las organizaciones y entre las organizaciones mismas y también desde el sistema social.

En síntesis, ante las diferencias que suelen surgir en todo colectivo humano en el que las organizaciones son una forma moderna de ellos, la transparencia (entendida como lo contrario a la corrupción) es una prerrogativa para acudir al diálogo y a la compresión de situaciones. Solo mediante la transparencia se podrá apelar a la escucha y a la comprensión pacífica de los problemas. La falta de transparencia elimina la posibilidad del diálogo. La falta de diálogo ocasionará conflictos no resueltos en los que la posibilidad de la violencia queda latente. Esta violencia buscará expresarse de una u otra manera, trastocando la paz y la sostenibilidad del sistema organizacional.

\section{Transformación organizacional hacia la transparencia}

El logro de objetivos públicos o privados implica un conjunto de complicados engranajes (Crozier y Friedberg, 1990). Detrás de cada objetivo conjunto hay un cúmulo de grupos de interés que afecta y es afectado por las decisiones. Más aún, detrás de cada actor institucional hay una diversidad de intereses difíciles de converger; de ahí que la transparencia es un principio fundamental de toda organización.

La sostenibilidad de una empresa hoy está relacionada con la capacidad que tiene para comunicar e integrar personas y grupos de trabajo en este complicado sistema de engranajes que se relacionan con la acción colaborativa sustentada en la transparencia. Asimismo, la sostenibilidad de una empresa está relacionada con la capacidad de comunicar e integrar actores externos en procesos de acción colaborativa para los que la transparencia es fundamental (Gilli, 2017).

La visión colaborativa y transparente hacia adentro y hacia fuera de las organizaciones requiere una transformación en la manera de pensar y actuar. Es necesario ir más allá de discursos aislados, se requiere congruencia entre palabras y acciones en el liderazgo del día a día para poder transmitir una visión común a los colaboradores (Arredondo, De la Garza y Vázquez, 2015). Se requiere de un entendimiento común que esté relacionado con el diseño de un futuro deseable para todas las partes y una claridad en la manera en que el trabajo de cada una de las partes sumará a esta visión. Así, la transparencia favorece la comunicación de información relevante y facilita las relaciones de integración y colaboración que demanda el entorno social.

Como ya hemos visto, la transformación organizacional de las empresas depende de una arquitectura de colaboración interna y externa. Esta colaboración implica beneficios y costos que se deben administrar (tabla 1).

Tabla 1. Beneficios y costos de la colaboración

\section{Beneficio de colaboración}

Recursos: potenciar recursos, no solo los económicos sino aquellos activos tangibles o intangibles que cada actor pueda proveer.

Productividad: administrar las ventajas competitivas que distintos actores tienen entre sí.

Información: el capital intelectual y de datos que cada actor pueda aportar desde su posición.

Legitimación: la colaboración de actores diferenciados puede dar la señal de legitimidad al público externo.

\section{Costos de colaboración}

Costos de proceso colaborativo: poner a los distintos actores de acuerdo requiere una inversión adicional de tiempo y esfuerzo.

Capacidad disminuida: en la virtud de integrar externos con ventajas competitivas, el espectro de producción propia tenderá a limitarse.

Control diluido: la autoridad y autonomía en definir objetivos y medios requiere de un proceso compartido en la negociación de acuerdos.

Reputación vulnerable: al colaborar existe un vínculo con actores externos a los cuales la organización queda ligada tanto en lo positivo como en lo negativo.

Fuente: Donahue y Zeckhauser (2006). 
Ante la demanda por una transformación organizacional, la colaboración precisa de asumir la responsabilidad de administrar los costos y riesgos implicados de manera proactiva. La transparencia juega un rol importante en la administración de estos costos y beneficios de la colaboración con actores que aseguren la sostenibilidad del negocio (Donahue and Zeckhauser, 2006).

Para la administración de los procesos colaborativos, es necesario especificar de manera clara y transparente aquellas dimensiones fundamentales que facilitan relaciones de colaboración exitosas con los distintos actores. Según Donahue y Zeckhauser (2006) esos elementos son la formalidad, la temporalidad de los acuerdos, el alcance de la colaboración, la diversidad de los participantes, la estabilidad de los procesos y la claridad en las responsabilidades de cada autoridad.

La sostenibilidad de la empresa está relacionada con la sostenibilidad de la sociedad, y en ambas la colaboración es indispensable. Para ello son necesarios los liderazgos transformacionales que guíen a la empresa a un nivel de integridad sin contradicción con el bien común (Arredondo and Maldonado, 2010). Hoy en día, empresa y sociedad no pueden subsistir de forma aislada. Empresa y sociedad, ambas con un enfoque hacia el bien común, facilitan la integración de intereses compartidos y con ello la pretensión de paz y sostenibilidad.

\section{La vía intersectorial: la empresa y el orden público para la paz y la sostenibilidad}

Es indiscutible que ante las actuales condiciones de interdependencia, el sector empresarial haya evolucionado su rol protagónico en lo económico para convertirse en una entidad estratégica en lo político y lo social. En un informe reciente de las Naciones Unidas se presenta a la empresa no solo como responsable de no causar daños y aportar a la filantropía, sino también como un aliado clave para alcanzar los nuevos Objetivos de Desarrollo Sostenible (Navazo, 2015).

En el documento Perspectivas y aportes empresariales para la construcción de paz de la Cámara de Comercio de Bogotá, la Fundación Ideas para la Paz y el Instituto Catalán Internacional para la Paz (2015), se asegura que el sector empresarial es un aliado clave para la sostenibilidad de la paz. Propone tres líneas estratégicas de aportación empresarial: (i) contar con lineamientos claros en cuanto a la debida diligencia y acción sin daño; (ii) desarrollar alianzas público-privadas que contribuyan al desarrollo local y la ampliación de la democracia y (iii) emprender acciones encaminadas a la reconciliación.

El mismo documento, refiere a los empresarios como un complemento ideal al sector académico en la integración de la sociedad a los temas públicos. Los empresarios aportan su capacidad de actuar y lo académicos aportan la reflexión y el conocimiento. El trabajo conjunto permite la generación de un conocimiento que favorezca la acción correcta.

Considerando este sentido de interdependencia, es necesaria la realización de esfuerzos intencionados y sistémicos para el logro de objetivos compartidos en los que la transparencia es básica para lograrlos. La sostenibilidad de la empresa depende de la sostenibilidad de su entorno y en la medida de que este entorno es complejo de forma creciente, la empresa tiene la responsabilidad ineludible de integrarse al orden de lo público; y desde su responsabilidad, sumar a los proyectos que promuevan la paz y la sostenibilidad de las comunidades (Schauer, 2014).

Estos proyectos, si no están soportados en la transparencia difícilmente podrán sostenerse. La integración de las empresas a las redes de colaboración en la construcción de paz y sostenibilidad implica un grado de apertura que permita una coexistencia con actores que, en medio de las evidentes diferencias, compartan objetivos comunes (Webel and Galtung, 2007).

Kooiman (1993) pone de manifiesto que las recientes modificaciones de los sistemas de interacción entre el sector público y el privado están relacionadas con la naturaleza compleja y dinámica de los sistemas sociopolíticos en los que nos desenvolvemos.

La complejidad y el dinamismo se suman a la torpeza burocrática, a la ineficiencia directiva de los gobiernos en el pasado reciente. Sus efectos 
se han evidenciado en crisis fiscales, políticas y económicas. Existe un descontento social ante la incapacidad de adaptación del gobierno a variables exógenas como la globalización y la apertura comercial, los altos niveles de corrupción, la polarización social y la irresponsabilidad de la administración pública entre otras. La autoridad y capacidad de quienes ocupan las jerarquías gubernamentales han pasado a ser insuficientes. Hay nuevas demandas en la gestión de lo público; demandas que generan la necesidad de redes amplias de participantes independientes pero con intereses públicos que los ligan, permitiendo el compromiso y el involucramiento para cubrirlas (Radin, 2007).

Resulta evidente el porqué de las formas emergentes de gobernar y sus modificaciones a los sistemas tradicionales. La visión de un Estado vertical ha ido poco a poco dando paso a una concepción cada más horizontal. Los actores sociales han ido teniendo cada vez más influencia en la política y en la administración al grado de llegar a niveles nunca antes vistos (Peter y Pierre, 2005).

De acuerdo con Cunnill (2005) la noción de una participación intersectorial remite en principio a la integración de diversos sectores con vistas a la solución de problemas sociales. La participación intersectorial puede ser definida como la convergencia de esfuerzos de diferentes sectores gubernamentales y no gubernamentales para producir políticas integrales e integradas que ofrezcan respuestas a las necesidades comunes. También puede definirse como la articulación de saberes y experiencias en el planeamiento, realización y evaluación de acciones con el objetivo de alcanzar resultados integrados en situaciones complejas que buscan un efecto sinérgico en el desarrollo social. La intersectorialidad puede allanar el camino para el trabajo interdisciplinario y transectorial a partir de los siguientes elementos que lo caracterizan: (i) integración de objetivos, integración administrativa y de procesos; (ii) inclusividad desde el diseño hasta la evaluación de las acciones; (iii) mancomunidad dado que supone compartir recursos, responsabilidades y acciones (Cunnill, 2005).

La intersectorialidad debe permitir mejoras significativas en el rendimiento de los programas ejecutados y generar una nueva perspectiva de entendimiento de conceptos como la paz y la sostenibilidad.

Los beneficios de este tipo de colaboración son relevantes gracias al acceso compartido de los recursos en red. El gobierno tiene capacidades que pueden beneficiar al sector empresarial y viceversa, y lo mismo aplica para la sociedad civil organizada. El beneficio particular de los participantes (incluido el gobierno) colabora en un beneficio colectivo, en el entendimiento de que los miembros de la red comparten objetivos y metas en común (Jarstad and Belloni, 2012).

Kettl (2009) propone el término de redes de gobierno a través de dos distintas vertientes: (i) como una descripción empírica al entretejido de estrategias y tácticas que usan los miembros de la red para coordinar sus acciones; (ii) como una prescripción normativa para desarrollar las estrategias que permitan suplir de la mejor manera las demandas públicas. Rhodes y Marsh (1994) la definen como un complejo de organizaciones relacionadas entre sí, con dependencia de recursos y que se distinguen de otros grupos o complejos por la estructura de esta dependencia. Ruano (2002) argumenta que el concepto de las redes da respuesta, al menos parcialmente, a estas cuestiones. Propone un esquema de interpretación práctica de las nuevas relaciones Estado-sociedad, que son caracterizadas por una estructura más horizontal y por el carácter relativamente informal de los intercambios (incluyendo al sector privado). De esta manera el concepto de red intersectorial se basa en la interacción de empresas, organizaciones e instituciones interdependientes donde la dinámica empresarial emerge como un actor estratégico. Estas relaciones se adaptan a los contextos y forman parte de una perspectiva de las formas en las que estos actores interactúan (Ruano, 2002).

Si bien las democracias occidentales pudieron vivir sin la exigencia de transparencia y acceso a la información (Aguilera, 2010), con la entrada de modelos contemporáneos de redes de colaboración interinstitucional, la transparencia se ha vuelto un asunto de importancia fundamental tanto para el sector público como el privado y la sociedad civil organizada.

El desarrollo social, monitoreado por el rendimiento económico, requiere hoy de la integración de fenómenos de tipo cultural y moral. Colaborar 
es una cuestión de relaciones, pero las relaciones no se basan en cálculos racionales de costos y beneficios sino en una genuina preocupación y vulnerabilidad mutua (Senge et al., 2008). La transparencia permite, ante este escenario de vulnerabilidad, generar un ambiente de confianza (Cruz, 2015) que resulta particularmente apremiante en un contexto intersectorial.

Por último, la transparencia es un principio fundante a partir del cual se puede empezar a construir esta capacidad de colaboración interna a la organización o externa en la generación de redes de colaboración intersectorial para el bien común. Esta relación de dependencia intersectorial implica lidiar con dificultades que van desde la resistencia para generar cohesión social hasta la necesidad de generar mecanismos que hagan transparente la red de colaboración entre los sectores.

\section{El sector empresarial y la construcción de paz en una situación de posconflicto}

\section{Integración del sector empresarial en la construcción de paz}

En una situación de posconflicto, la firma de acuerdos por la paz es una oportunidad para optar por un trabajo de transformación social hacia una paz sostenible donde la participación empresarial es fundamental (Cámara de Comercio de Bogotá, Fundación Ideas para la Paz e Instituto Catalán Internacional para la Paz, 2015).

En el caso de Colombia, tras la firma del acuerdo de paz, diferentes actores estratégicos han alzado la voz para evidenciar la responsabilidad del sector privado como indelegable. El rol empresarial debe trascender de la generación de empleo y el pago de impuestos hacia una asociación con el gobierno para la creación de una visión pacífica y sostenible de la región que se pueda imaginar y construir de manera colaborativa. El sector empresarial puede aportar al fortalecimiento de las instituciones a partir de la generación de capacidades para la convivencia, de mercados inclusivos y la promoción del diálogo que permita nuevas formas de pensamiento y la visión de oportunidades para la sostenibilidad (Oetzel et al., 2009).

Esta visión empresarial por la paz no está libre de obstáculos, pues alienar intereses no es tarea fácil; especialmente cuando parte del empresariado se ha visto beneficiado de la situación de conflicto y falta de paz en la región. De igual manera el sector empresarial no siempre ve sus intereses afectados de forma directa por el conflicto y la falta de paz, por lo que se cuestiona la capacidad del sector para responder a una realidad que le puede resultar ajena (Annan, 2004). A pesar de las condiciones del conflicto, que pueden llegar a parecer insuperables, existe también la posibilidad de imaginar una región diferente con una economía en crecimiento y un tejido sólido que articule lo social, lo empresarial y lo público de forma sostenible. Por ello es necesario contar con líderes empresariales ampliamente comprometidos con la paz y la sostenibilidad. Es preciso que estos líderes sean promotores y ejemplo de diálogo ante el conflicto, y mediante ello logren la reconstrucción social para una paz sostenible (Navazo, 2015).

De este modo la sostenibilidad de una región en una etapa de posconflicto requiere de un trabajo armónico entre actores que se verá beneficiado por programas que fomenten la transparencia entre empresas, entre estas y el sector gubernamental y entre ellas y la sociedad civil organizada (Annan, 2004).

\section{Ejemplos de programas por la transparencia empresarial}

En este proceso intersectorial donde la confianza es básica, la transparencia juega un rol protagónico. A continuación, se presentan un par de programas para la transparencia empresarial. Ambos son ejemplos de acciones que aportan de manera activa e intencionada en la construcción de los fundamentos sobre los que las empresas pueden apoyar la transparencia y la legitimidad de las redes de colaboración por la paz y la sostenibilidad. 
1. Programa "Rumbo empresas-íntegras y transparentes".

Este programa es una herramienta de manejo gerencial que busca promover prácticas éticas en la gestión empresarial. Busca crear valor y confianza en las relaciones de negocio. Las características del programa son: (i) autogestionada, las empresas pueden aplicar la herramienta de manera autónoma y directa por medio de una serie de ejercicios prácticos propuestos para que los emplee según sus necesidades concretas; (ii) participativa, promueve dinámicas de trabajo que permiten que todos los miembros de la empresa se involucren en el proceso de construcción de la ética empresarial y lo interioricen; (iii) práctica, busca generar compromisos y consensos al hacer énfasis en la práctica más que en el discurso y al combinar la reflexión individual con la colectiva; (iv) flexible, en su metodología, con el fin de que cada empresa la adapte a su contexto y decida qué módulos de apoyo temático necesita trabajar y/o fortalecer de acuerdo con las necesidades propias de la empresa; (v) consistente, aunque cada uno de los módulos puede ser implementado independientemente todos están diseñados de forma tal que guarden una coherencia interna tanto en su enfoque como en su metodología.

Entre los beneficios empresariales que ofrece están: mejora la imagen corporativa; incrementa la productividad y la competitividad; incrementa la toma de decisiones con sentido ético; favorece la generación y la implementación de buenas prácticas empresariales; incentiva la rendición de cuentas y el sentido de pertenencia por la empresa y contribuye a un entorno de negocios más ético.

Este instrumento evidencia una intencionalidad y un método que facilita acciones de gestión interna para la promoción de la transparencia. Empresas que asuman la responsabilidad por la construcción de una cultura de legitimidad tienen la facilidad de tomar como referencia este tipo de programas y encontrar sus propias vías para lograr la paz y la sostenibilidad regional.

2. Programa "Medición de transparencia empresarial".

Este programa es una herramienta de control social que tiene como objetivo monitorear y evaluar mecanismos y políticas de transparencia empresarial. Tiene la intención de fortalecer al sector privado a través de la adopción de buenas prácticas de integridad a la hora de relacionarse con los grupos de interés con los que se organiza. El programa pretende ser un instrumento para el fortalecimiento institucional de la empresa y de su entorno de negocios.

Tabla 2. Componentes del programa "Medición de transparencia empresarial"

\begin{tabular}{|c|c|c|}
\hline Reglas claras & Integridad corporativa & Ética y gobierno \\
\hline \multirow[t]{7}{*}{ Apertura } & \multirow[t]{3}{*}{ Programa anticorrupción } & Sistemas de gestión \\
\hline & & Prevención \\
\hline & & Detección, investigación y sanción \\
\hline & Información socialmente útil & Grupos de interés primarios \\
\hline & \multirow[t]{3}{*}{ Transparencia activa } & Grupos de interés secundarios \\
\hline & & Criterios de accesibilidad \\
\hline & & Evaluación de pertinencia \\
\hline \multirow[t]{4}{*}{ Diálogo } & \multirow[t]{2}{*}{ Gestión de relacionamiento } & Canales de diálogo entre grupos de interés \\
\hline & & Rendición de cuentas \\
\hline & \multirow[t]{2}{*}{ Derecho de acceso a la información } & Gestión de requerimientos \\
\hline & & Seguimiento al relacionamiento \\
\hline \multirow[t]{4}{*}{ Control } & \multirow[t]{2}{*}{ Controles internos } & Auditoría \\
\hline & & Cumplimiento \\
\hline & \multirow[t]{2}{*}{ Promoción del control social } & Control social \\
\hline & & Ciudadanía corporativa \\
\hline
\end{tabular}


Los beneficios para las empresas que apuestan por la transparencia son los siguientes: fortalece su sistema de integridad corporativa, lo que a la vez se traduce en confianza en la relación con sus grupos de interés; incrementa el orgullo por su empresa y la buena reputación de la misma; le representa una ventaja competitiva que se refleja en los resultados sociales y financieros de la empresa; fortalece la lealtad y el compromiso de su recurso más importante, sus colaboradores; fortalece su capacidad para monitorear, prevenir y gestionar riesgos de corrupción; le permite a la empresa compararse con los más altos estándares y principios internacionales en transparencia e integridad; le permite identificar oportunidades de mejora y le ayuda a traducirlas en ventajas estratégicas y operacionales.

La relevancia de este instrumento, es que facilita un método de evaluación interna que permite procesos de mejora continua en la cultura de transparencia empresarial. Como herramienta permite un método para monitorear los progresos de transformación hacia una organización más transparente.

\section{Ejemplos de red de colaboración por la paz en Colombia}

El valor que tienen las redes de colaboración en la legitimización de proyectos para la construcción de paz y sostenibilidad queda manifestado en iniciativas que promueven la participación de distintos actores sociales en las intenciones de paz y sostenibilidad. A continuación, se presentan un par de iniciativas que ejemplifican los resultados de este tipo de esfuerzos colaborativos.

1. Iniciativa "Desarrollo de una estrategia de trabajo en la construcción de paz desde el sector empresarial en Colombia".

Esta iniciativa integra al Consejo Internacional de la Industria Sueca -NIR-, la Asociación Nacional de Empresarios de Colombia -ANDI- y la Fundación Ideas para la Paz -FIP- en un proyecto orientado a superar el conflicto y a aportar en la construcción de una paz sostenible desde el sector empresarial en Colombia.
En una primera fase se realizó un diagnóstico que permitió la sensibilización respecto a la necesidad de estrategias y prácticas que se necesitan integrar en la gestión empresarial, en la superación del conflicto y en la construcción de una paz sostenible. Para esta etapa se llevaron a cabo talleres de diagnóstico regional para la identificación de los problemas estratégicos a trabajar. Los problemas identificados y sus respectivas propuestas de trabajo quedaron de la siguiente manera: (i) seguridad ciudadana, se propone un programa de trabajo que fomente la cultura de la prevención y los mecanismos de resolución de conflictos alternos; (ii) corrupción (soborno y extorsión), se propone un programa de trabajo en alianza entre empresas-autoridades que funcione hacia el interior de las empresas; (iii) operación empresarial en entornos complejos, se propone la diversificación de sectores económicos e inversión social.

A esta etapa de diagnóstico siguió una etapa de diseño de estrategias conjuntas de trabajo, en donde se implantaron las recomendaciones generadas como propuesta de trabajo a los problemas específicos. Cabe mencionar que durante este proceso el Observatorio de Responsabilidad Social Empresarial pretende funcionar como una herramienta para la transparencia. Se busca que los empresarios y grupos de interés accedan a información relevante en temas de empresa, derechos humanos y construcción de paz.

En esta iniciativa no solo se puede ver el funcionamiento de una red de colaboración intersectorial sino un esfuerzo intencionado en la implementación de estructuras que faciliten la comunicación y transparencia entre los distintos actores sociales.

2. Iniciativa "Perspectivas y aportes empresariales para la construcción de paz".

Esta iniciativa integró a la FIP, al Instituto Catalán Internacional para la Paz y a la Cámara de Comercio de Bogotá. La alianza recogió las percepciones de los distintos sectores frente a las tareas que cada uno debe asumir en la construcción de una Colombia sostenible y en paz.

A partir del ejercicio consultivo se identificaron cuatro líneas de acción para desarrollar un proceso conjunto de construcción de paz: (i) línea de acción política: emprender acciones que 
priorizan el diálogo y la participación de múltiples actores, fomentan iniciativas en reconciliación e incluyen sectores de la población históricamente excluidos; (ii) línea de acción económica: desarrollar acciones encaminadas a la generación de ingresos para poblaciones vulnerables y la reintegración socioeconómica de excombatientes; (iii) línea de acción sociocultural: impulsar iniciativas en educación, valores comunitarios y sentidos de pertenencia; (iv) línea de acción gobernanza: promover el fortalecimiento institucional, la articulación entre el ámbito nacional y local en las estrategias de construcción de paz y garantizar el acceso a la verdad, la justicia, la reparación y las garantías de no repetición en todos los territorios (Cámara de Comercio de Bogotá, Fundación Ideas para la Paz e Instituto Catalán Internacional para la Paz, 2015).

Esta iniciativa integra un proceso de comunicación intersectorial y representa un marco inicial de comunicación con el sector empresarial para la definición de roles y actividades específicas en la construcción de paz.

Como puede constatarse hay esfuerzos específicos orientados hacia la transparencia empresarial para consolidar la paz y la sostenibilidad en la región, los cuales son aplicados a Colombia.

Las experiencias empresariales a favor de la paz presentan beneficios directos: un quehacer organizacional, logrando mayor eficiencia; un mayor compromiso por parte de los colaboradores y una mejora en relación a los diversos agentes con los que vincula su actividad (Jarstad and Belloni, 2012). Por otra parte, los esfuerzos intersectoriales coinciden en orientar acciones hacia tres estrategias puntuales: educar en el diálogo para la resolución de conflictos; fortalecimiento institucional y trabajar en iniciativas de inclusión social para sectores vulnerables.

\section{Conclusiones}

El objetivo de este artículo ha sido abordar la vinculación de la corrupción empresarial en los temas de paz y sostenibilidad, la necesidad de un proceso transformacional en las organizaciones en aras de una mayor transparencia y la necesidad de ejercer esfuerzos intersectoriales entre el gobierno, la empresa y la sociedad civil organizada para lograr mejores condiciones de paz y garantizar la sostenibilidad. También se refirió al rol que tienen las empresas en una situación posconflicto, así como algunos ejemplos de programas por la transparencia empresarial y la colaboración intersectorial. Se buscó con ello ofrecer algunas posibles vías de acción, asumiendo que cada región tendrá que crear espacios para lograr consensos y acuerdos orientados a la paz.

Finalmente se concluye que en un mundo cada vez más global e interconectado, todos los actores de la sociedad tienen responsabilidad en la construcción de un futuro sostenible. Se necesitan nuevas formas de pensar y actuar a la hora de dar cara a los problemas sociales. Se requiere un cambio cultural que facilite una visión donde los valores como la transparencia y la colaboración sean fundamentos, y a partir de los cuales sea posible construir nuevos escenarios. No hay camino viable que ignore las necesidades de generaciones futuras. La paz en una sociedad compleja parte de una sostenibilidad basada en redes de colaboración institucional alineadas y transparentes que faciliten el logro de objetivos compartidos. El poder repensar una organización como debería de ser requiere imaginarnos la vida empresarial y sus actores con los que se vincula de otra manera, en otro momento y en otra realidad. La imaginación para la paz y la sostenibilidad no es ilusión. Implica proyectar la realidad a situaciones superiores y mejores; se trata de llevar lo imaginado a lo realizable, pensar que otro mundo es posible.

\section{Referencias}

Aguilera, J. (2010). Transparencia y democracia: claves para el concierto. Cuadernos de Transparencia, 10. Recuperado de http://www.resi.org.mx/icainew_f/images/Biblioteca/Cuaderno\%20transparencia/cuadernillo10.pdf.

Albu, O. and Flycerbom, M. (2016). Organizational transparency: Conceptualizations, conditions and consequences. Business \& Society, 4, 1-30.

Alejos, C. (2015). Transparencia: fuente de confianza en la empresa. Cuadernos de la Cátedra "la Caixa" de Responsabilidad Social de la Empresa y Gobierno 
Corporativo, 27. Recuperado de http://www.iese. edu/Aplicaciones/upload/catedralacaixavol27. pdf.

Alonso, M. (2009). La transparencia de las empresas en internet para la confianza de los accionistas e inversoras: un análisis empírico. Cuadernos de Administración, 22 (38), 11-30.

Annan, K. (2004). The causes of conflict and the promotion of durable peace and sustainable development in Africa. African Renaissance, 14 (2), 9-42.

Arredondo, F. and Maldonado, V. (2010). Differences between the relationship of Integrity and leadership styles according to the Model of Bernard Bass. Estudios Gerenciales, 26 (114), 35-48.

Arredondo, F., De la Garza, J. y Vázquez, J. (2015). Transparencia y rendición de cuentas en las organizaciones, basado en un modelo estructural. En XX Congreso Internacional de Contaduría, Administración e Informática, UNAM, Ciudad Universitaria, México.

Arredondo-Trapero, F., De la Garza-García, J. y Villa-Castaño, L. (2015). Colaboración organizacional desde la perspectiva del empleado: caso de las maquiladoras del noreste de México. Revista Venezolana de Gerencia, 20 (72), 698-716.

Bandsuch, M., Pate, L. and Thies, J. (2008). Rebuilding Stakeholder Trust in Business: An Examination of Principle-Centered Leadership and Organizational Transparency in Corporate Governance. Business and Society Review, 113 (1), 99-127.

Briano, G. y Saavedra, M. (2015). La composición del consejo de administración y la estructura accionaria como factores explicativos de la transparencia en el gobierno corporativo en Latinoamérica: evidencia en empresas cotizadas de Argentina, Brasil, Chile y México. Estudios Gerenciales, 31 (136), 275-286.

Cámara de Comercio de Bogotá, Fundación Ideas para la Paz e Instituto Catalán Internacional para la Paz. (2015). Perspectivas y aportes empresariales para la construcción de paz. Bogotá, Colombia: FIP.

Castañeda, V. (2016). Una investigación sobre la corrupción pública y sus determinantes. Revista Mexicana de Ciencias Políticas y Sociales, LXI (227), 103-135.
Christensen, L. and Cornelissen, J. (2015). Organizational transparency as myth and metaphor. European Journal of Social Theory, 18 (2), 132-149.

Ciamberlani, L. y Steinberg, L. (1999). Comunicación para la transparencia. Buenos Aires, Argentina: Ediciones Granica.

Crozier, M. y Friedberg, E. (1990). El actor y el sistema: las restricciones de la acción colectiva. Ciudad de México, México: Alianza.

Cruz, J. (2015). Moral y transparencia. Fundamentos e implicaciones morales de la transparencia. Cuadernos de Transparencia, 15. Recuperado de http:// inicio.ifai.org.mx/Publicaciones/Cuadernillo\%20 15\%20B.pdf.

Cuevas, R. (2005). La construcción de la ética de la empresa o ética de los negocios. Contaduría y Administración, 217, 119-142.

Cunill, N. (2005). La intersectorialidad en el gobierno y gestión de la política social. En X Congreso Internacional del CLAD sobre la reforma del Estado y de la Administración Pública, Santiago de Chile, Chile.

del Campo, P., Herrador, T. y Segovia, A. (2016). La transparencia organizativa y económica en la Web de las fundaciones: un estudio empírico para España. Revista de Estudios Cooperativos, 121, 62-88.

Donahue, J. and Zeckhauser, R. (2006). Public-Private Colaboration. En Moran, M., Rein, M. and Goodin, R. (Ed.), The Oxford Handbook of Public Policy (pp. 496-524). Oxford, United Kingdom: Oxford University Press.

Etkin, J. (2007). Capital social y valores en la organización sustentable. Buenos Aires, Argentina: Ediciones Granica.

Galtung, J. (1996). Peace by peaceful means. Peace and conflict, development and civilization. London, England: SAGE.

Gilli, J. (2017). La transparencia como objetivo del desarrollo sostenible. Ciencias Administrativas, 9, 43-49.

Giménez, L. (2012). Transparencia y derechos laborales. Cuadernos de Transparencia, 12. Recuperado de http://inicio.inai.org.mx/Publicaciones/Cuadernillo\%2012\%20B.pdf. 
Guerrero, E. (2008). Para entender la transparencia. Ciudad de México, México: Nostra Ediciones.

Heimstädt, M. (2017). Openwashing: A decoupling perspective on organizational transparency. Technological Forecasting and Social Change, 125, 77-86.

Institute for Economics and Peace. (2015). Peace and Corruption. Recuperado de http://economicsandpeace.org/wp-content/uploads/2015/06/Peace-and-Corruption.pdf.

Institute for Economics and Peace. (2016). Positive Peace Report. Recuperado de http://visionofhumanity.org/sites/default/files/Positive\%20 Peace\%20Report\%202016.pdf.

Jarstad, A. and Belloni, R. (2012). Introducing hybrid peace governance: Impact and prospects of liberal peacebuilding. Global Governance, 18 (1), 1-6.

Kettl, D. (2009). The key to networked government. En Goldsmith, S. and Kettl, D. (Ed.), Unlocking the power of Networks: Keys to high-performance government (pp. 1-14). Washington, USA: Brooking Institution Press.

Kooiman, J. (Dir.) (1993). Modern governance. New government-society interactions. London, England: SAGE.

Kotter, J. (1996). Leading change. Boston, USA: Harvard Business School Press.

Lindley, D. (2007). Promoting peace with information: Transparency as a tool of security regimes. New Jersey, USA: Princeton University Press.

Manfredi, J., Herranz, J. y Calvo, L. (2017). Transparencia y diplomacia: nuevas demandas sociales y rutinas profesionales. Revista Latina de Comunicación Social, 72 (8), 832-848.

Marcuello, C. et al. (2007). Transparencia y rendición de cuentas en las empresas de inserción. CIRIEC-España. Revista de Economía Pública, Social y Cooperativa, 59, 91-122.

Navazo, P. (2015). El sector empresarial en Colombia, nuevo actor para la paz. Recuperado de http://www.esglobal.org/colombia-camino-a-la-paz-con-el-sector-empresarial/.

Oetzel, J. et al. (2009). Business and peace: Sketching the terrain. Journal of Business Ethics, 89 (4), 351373.
Pérez, R. (2016). Corrupción y costos de transacción en la administración pública. Revista Iberoamericana de las Ciencias Sociales y Humanísticas, 5 (10), 188-213.

Peter, G. y Pierre, J. (2005). ¿Gobernanza sin gobierno? Replanteándose la administración pública. En Cerrillo, A. (Coord.), La gobernanza hoy: 10 textos de referencia (pp. 123-144). Madrid, España: Instituto Nacional de Administración Pública.

Radin, B. (2007). The instruments of intergovernmental management. En Peter, G. and Pierre, J. (Ed.), Handbook of Public Administration (pp. 365-376). London, England: SAGE.

Rawlins, B. (2008). Give the emperor a mirror: Toward developing a stakeholder measurement of organizational transparency. Journal of Public Relations Research, 21 (1), 71-99.

Reyes Heroles, F. (2007). Corrupción: de los ángeles a los índices. Cuadernos de Transparencia, 1. Recuperado de https://www.tm.org.mx/corrupcion-de-los-angeles-a-los-indices/.

Rhodes, R.A.W. and Marsh, D. (1994). Policy networks: 'defensive' comments, modest claims and plausible research strategies. En Political Studies Association, Annual Conference, University of Swansea, Swansea, United Kingdom.

Ruano, J. (2002). La gobernanza como forma de acción pública y como concepto analítico. En VII Congreso Internacional del CLAD sobre la reforma del Estado y de la administración pública, Lisboa, Portugal.

Schauer, F. (2014). Transparencia en tres dimensiones. Revista de Derecho, XXVII (1), 81-103.

Schnackenberg, A. and Tomlinson, E. (2016). Organizational transparency: A new perspective on managing trust in organization-stakeholders relationships. Journal of Management, 42 (7), 1784-1810.

Senge, P. et al. (2008). The necessary revolution. How individuals and organizations are working together to create a sustainable world. New York, USA: Doubleday.

Spreitzer, G. (2007). Giving peace a chance: Organizational leadership, empowerment, and peace. Journal of Organizational Behavior: The International Journal of Industrial, Occupational and Organizational Psychology and Behavior, 28 (8), 1077-1095. 
Socias, A. y Horrach, P. (2013). Enfoque de la responsabilidad social y la transparencia en empresas de economía solidaria. CIRIEC-España. Revista de Economía Pública, Social y Cooperativa, 77, 31-58.

Testera, A. y Cabeza, L. (2013). Análisis de los factores determinantes de la transparencia en RSC en las empresas españolas cotizadas. Intangible Capital, $9(1), 225-261$.

Uvalle, R. (2007). Gobernabilidad, transparencia y reconstrucción del Estado. Convergencia. Revista de Ciencias Sociales, 14 (45), 47-74.

Uvalle, R. (2016). Fundamentos de la transparencia en la sociedad contemporánea. Revista Mexicana de Ciencias Políticas y Sociales, LXI (226), 199-220.

Vergara, R. (2005). La transparencia como problema. Cuadernos de Transparencia, 5. Recuperado de http://inicio.inai.org.mx/Publicaciones/Cuadernillo\%2005\%20B.pdf.

Villarreal, R. y Villarreal, T. (2003). IFA: la empresa competitiva sustentable en la era del capital. Ciudad de México, México: McGraw-Hill.

Webel, C. and Galtung, J. (2007). Handbook of peace and conflict studies. New York, USA: Routledge. 